\title{
Backpressure Meets Taxes: Faithful Data Collection in Stochastic Mobile Phone Sensing Systems
}

\author{
Shusen Yang \\ Imperial College Lodon \\ Email: s.yang09@imperial.ac.uk
}

\author{
Usman Adeel \\ Imperial College London \\ Email: u.adeel09@imperial.ac.uk
}

\author{
Julie McCann \\ Imperial College London \\ Email: j.mccann@imperial.ac.uk
}

\begin{abstract}
The use of sensor-enabled smart phones is considered to be a promising solution to large-scale urban data collection. In current approaches to mobile phone sensing systems (MPSS), phones directly transmit their sensor readings through cellular radios to the server. However, this simple solution suffers from not only significant costs in terms of energy and mobile data usage, but also produces heavy traffic loads on bandwidth-limited cellular networks. To address this issue, this paper investigates cost-effective data collection solutions for MPSS using hybrid cellular and opportunistic short-range communications. We first develop an adaptive and distribute algorithm OptMPSS to maximize phone user financial rewards accounting for their costs across the MPSS. To incentivize phone users to participate, while not subverting the behavior of OptMPSS, we then propose BMT, the first algorithm that merges stochastic Lyapunov optimization with mechanism design theory. We show that our proven incentive compatible approaches achieve an asymptotically optimal gross profit for all phone users. Experiments with Android phones and trace-driven simulations verify our theoretical analysis and demonstrate that our approach manages to improve the system performance significantly (around $\mathbf{1 0 0 \%}$ ) while confirming that our system achieves incentive compatibility, individual rationality, and server profitability.
\end{abstract}

\section{INTRODUCTION}

Ubiquitous sensor-rich smartphones are beginning to play an increasingly important role in the evolution of the urban Internet of Things (IoTs), which bridge the digital space to the physical world at a societal scale. Their powerful computing and communication capacities, huge market proliferation, and inherent mobility makes Mobile Phone Sensing Systems (MPSS) [1] a much more flexible and cost-effective sensing solution compared with traditional static sensor networks. In turn this has motivated the design of novel sensing applications [1], [2], covering urban environmental monitoring [3], smart transportation [4], and safety [5].

Most of current MPSSs transmit mobile sensor data to the server through cellular networks. When MPSS becomes popular, this simple solution will suffer from not only significant battery [6], [7] and 3G/4G financial costs [8] to the phone users, but also will produce heavy traffic load on the underlying bandwidth-limited cellular networks, especially for MPSS applications that require continuous sensing with fine granularity (e.g. [9]).

Besides the expensive cellular communications, current smartphones are being equipped with more and more shortrange wireless technologies such as $\mathrm{WiFi}$, WiFi direct, and Bluetooth 4.0, which enable opportunistic phone-to-phone and phone-to-server communications (e.g. through WiFi routers). Due to their low energy and financial costs, it is promising to exploit the potential of short-range communications in MPSSs, especially for delay-tolerant mobile sensing applications [7]. For instance, without using its own cellular radio, a phone can report its sensor data to the server, through another proximity phone with a cheaper cellular cost (e.g. unlimited mobile data) or when it passes a free WiFi router. However, to build such a MPSS with hybrid cellular and short-range communications, the following research issues must be addressed:

Networking Issues. It is a challenge to perform the sensing and opportunistic multi-hop data transmission tasks that are adaptive to the time-varying and potentially unpredictable network states, including fluctuating wireless channel quality; intermittent connectivity caused by phone user movement; heterogeneous transmission and sensing costs across mobile phones; 3G/4G mobile data costs; and the opportunistic availability of nearby free Internet access points.

Economic Issues. To encourage the phone users to participate the MPSS, they should be properly rewarded to cover sensing and transmission costs [10]-[12]. In addition, the self-interest phone users may try to maximize their benefits strategically by misreporting their local state parameters. For instance, in order to prolong battery lifetime, a phone user may hide that she is connected to a free WiFi router to avoid relaying other nearby phone data to the server. This would result in significant performance degradation of the system. Therefore, incentivization is a key issue for MPSS with this new networking paradigm, which is much more challenging compared to pure cellular networks.

\section{A. Our Approach}

In this paper, we present theoretical and practical studies to address above two issues. Our contributions are summarized as follows:

1. We formulate a finite-horizon stochastic optimization problem for continuous data collection in MPSS using hybrid cellular and opportunistic short-range communications. The objective of the formulated problem is to maximize the global gross profit, i.e. the total financial rewards of all phone users after costs incurred by performing the sensing and transmission tasks are deducted.

2. We develop a lightweight joint sensing rate control and dynamic routing algorithm, OptMPSS to solves the data 
collection problem in a fully distributed and therefore scalable way. We prove that OptMPSS is an asymptotically optimal solution to the formulated problem.

3. We propose a fully distributed mechanism, Backpressure Meet Taxes (BMT), to incentivize phone users to faithfully implement OptMPSS, by imposing taxes or providing subsidies for each phone user, depending on her impact on the rest of phone users in the MPSS. We prove that BMT manages to achieve asymptotic incentive compatibility [13]. To our knowledge, BMT is the first approach that integrating algorithmic mechanism design theory [14], [15] to the stochastic Lynapunov optimization framework [16]. Besides MPSS, this method developed for BMT also has a great potential to be applies to other stochastic distributed systems with self-interest and strategic users.

4. Through experiments with WiFi-direct-enabled Android devices and extensive simulations with real human mobility trace [17], we demonstrate that system performance can be significantly improved by exploiting low-cost short-range communications, in terms of global social profits and phone users' costs. Evaluation results also show that each phone user can always get a positive net profit (i.e. gross profit plus subsidies or minus taxes) and the server never incurs a deficit (i.e. the server always obtain a positive profit). Furthermore, each phone user cannot increase her net profit improvement by lying about her private parameters. These results demonstrate that BMT can achieve individual rationality, server profitability, and incentive compatibility (faithful implementation) in practice.

\section{B. Related Work}

Recently, several incentive-based mechanisms have been proposed for MPSS [10]-[12], [18]. [11] develops platformcentric and user-centric schemes based on a Stackelbergy game and auction theory respectively. [12] proposes a mechanism based on a Bayesian game to minimize participation costs while ensuring certain service qualities, by determining the level of user participation (i.e. sensing rate). However, all of these schemes focus on MPSS with pure cellular radios only, which cannot be directly used in MPSS with hybrid cellular and multi-hop short-range communications.

The explosive growth of cellular traffic has motivated an increase in research into cellular traffic offload using other forms of opportunistic connectivity, including WiFi [19]-[21] and Bluetooth [22]. However, none of these focus on MPSS. EffSense [7], considers MPSS with the same hybrid wireless networks as us. However, this heuristic-based scheme does not provide any performance guarantees, and does not consider incentivisation for the strategic and self-interest phone users.

Stochastic Lyapunov optimization [16], [23]-[26] provides elegant and powerful theoretical tools to derive backpressure style cross-layer network optimization and control algorithms. Due to their adaptiveness to network dynamics, several backpressure rate control and routing schemes [23]-[25] have been proposed for opportunistic mobile networks. However,

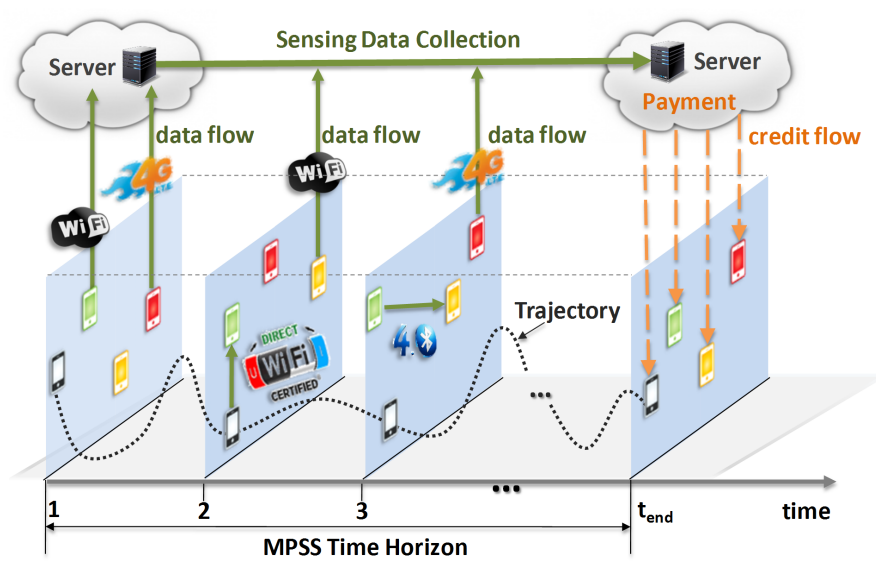

Fig. 1. An illustrative example of a MPSS with hybrid cellular and short-range communications, consisting of 4 phones and a server.

again none of them focus on MPSS nor do they account for incentivisation and the strategic behaviors of phone users.

Mechanism design [14], [15] is concerned with how to make a global decision with desirable properties in systems consisting of strategic self-interest individuals who have private information. Recent theoretical work [13], [27] on distributed Vickrey-Clarke-Groves (VCG) mechanisms enables the faithful implementation of algorithms producing desired outcomes (such as dual decomposition and average consensus) in a distributed way. However, these approaches focus on deterministic rather than stochastic systems. Our work combines threads of all the above works in a novel way.

\section{Paper Organization}

The next section presents the system model and design objectives. Section III describes the OptMPSS algorithm. Mechanism design models are established in Section IV. Section $\mathrm{V}$ presents the BMT algorithm, then Section VI discusses the performance evaluation. Finally, we conclude the paper in Section VII.

\section{System Model AND ObJectives}

As shown in Fig. 1, we consider a MPSS that consists of a server $S$ and a set of mobile phones $\mathcal{N}$ collecting urban sensing data. The MPSS operates during a finite time horizon (e.g. a week) with discrete time slots $t=\left\{1,2, \ldots, \mathrm{t}_{\text {end }}\right\}, \mathrm{t}_{\text {end }}<$ $+\infty$. Every phone can communicate with the server $S$ through $3 \mathrm{G} / 4 \mathrm{G}$ cellular radios, or through the low-cost WiFi when it passes a WiFi router (slots 1-3 in Fig. 1). In addition, phones in immediate proximity can communicate with each other, using short-range communications such as WiFi direct (slot 2) and Bluetooth 4.0 (slot 3).

\section{A. Sensing and Communication Models}

At each time slot $t$, each phone $i$ produces $0 \leq r_{i}(t) \leq$ $r_{\max }$ sensor data packets, where the finite sensing rate upper bound $r_{\max } \leq \infty$ is defined by the specific mobile sensing application. Let $\mu_{i, j}(t) \geq 0$ be the channel capacity from a phone $i \in \mathcal{N}$ to a phone $j \in \mathcal{N}$ or to the server $j=S$ 
at slot $t$, i.e. the maximum (integer) number of data packets that can be successfully transmitted from $i$ to $j$ during slot $t$. It can be seen that $\mu_{i, j}(t)$ may vary significantly over the time slots, due to the stochastic phone user movement and wireless channel qualities. In practice, all possible channel capacities (i.e. $\mu_{i, j}(t), \forall i, j, t$ ) must have an finite upper bound $\mu^{\max } \leq \infty$, determined by the finite data rate of the wireless transceivers .

Each phone $i$ maintains a time-varying temporary neighbor table $\mathcal{N}_{i}(t)$, consisting of the server $S$ (if currently connected) and the phones in proximity at slot $t$ :

$$
\mathcal{N}_{i}(t):=\left\{j: j \in \mathcal{N} \cup\{S\}, \mu_{i, j}(t)>0\right\}
$$

In practice, $\mathcal{N}_{i}(t)$ can be established by using neighbor discovery schemes such as [28]. Denote $0 \leq f_{i, j}(t) \leq \mu_{i, j}(t)$ as the amount of forwarded data from phone $i$ to its current neighbor $j \in \mathcal{N}_{i}(t)$ at slot $t$. We use a vector

$$
\boldsymbol{x}_{i}(t)=\left(r_{i}(t), f_{i, j}(t), j \in \mathcal{N}_{i}(t)\right)
$$

to represent the sensing and data forwarding actions of a phone $i \in \mathcal{N}$ at slot $t$

\section{B. Quеие Dynamics}

Each phone $i$ maintains a data queue with size $Q_{i}(t) \geq 0$ to store the sensing data collected by itself and received from other phones. Considering sensing and data forwarding dynamics, the queue backlog of each phone $i$ updates as follows:

$$
Q_{i}(t+1)=\left|Q_{i}(t)-f_{i}^{\text {out }}(t)\right|_{+}+r_{i}(t)+f_{i}^{\text {in }}(t)
$$

where for any real number $a$, the operator $|a|_{+}=a$ if $a>0 ;|a|_{+}=0$ otherwise. $f_{i}^{\text {out }}(t)=\sum_{j \in \mathcal{N}_{i}(t)} f_{i, j}(t)$, and $f_{i}^{i n}(t)=\sum_{j \in \mathcal{N}_{i}(t)} f_{j, i}(t)$ represent the total numbers of outgoing and incoming packets of phone $i$ at slot $t$ respectively. It is worth noting that the queue backlog of the server $S$ is always zero, i.e. $Q_{S}(t)=0, \forall t$, since it is the destination of all sensor data packets.

\section{Costs of Phones}

At each slot $t$, each phone incurs costs due to sensing and data transmission. Let $p_{i}^{s}(t)>0$ be the per packet sensing price of phone $i$ at slot $t$. Therefore, the sensing cost of phone $i$ at slot $t$ is $p_{i}^{s}(t) r_{i}(t)$. In practice, the sensing price $p_{i}^{s}(t)$ depends on both MPSS application requirements, and the available resources that phone $i$ has at time $t$ such as remaining battery energy. Denote $p_{i, j}^{t}(t)$ as the per packet transmission price for phone $i$ to send a sensor data packet to a temporary neighbor $j$. If $j$ is the server $S$, then $p_{i, j}^{t}(t)$ depends on financial cellular costs, the availability of a nearby WiFi router, and the remaining battery level of phone $i$. Specifically, price $p_{i, j}^{t}(t)$ are normally significantly smaller when $i$ sends data to the server through WiFi than through cellular radios. If $j$ is another phone, the transmission price $p_{i, j}^{t}(t)$ mainly depends on battery concerns of the users of phones $i$ and $j$. We can see that the data transmission price is highly dynamic and heterogeneous across different wireless transmission links.
It is worth noting that although sensing and transmission prices are influenced by various practical aspects, they can be all normalized to monetary values (e.g. US dollar or credits per packet), estimated by each phone user herself rather then the server. The detailed estimation in practice is out of the scope of this paper, but our BMT scheme can guarantee that faithful estimation is the best strategy for each self-interest phone user.

For a given slot $t$, the total cost of each phone $i \in \mathcal{N}$ can be computed as

$$
\operatorname{cost}_{i}(t)=\boldsymbol{p}_{i}(t) \boldsymbol{x}_{i}^{\top}(t)
$$

where the vector

$$
\boldsymbol{p}_{i}(t)=\left(p_{i}^{s}(t), p_{i, j}^{t}(t), j \in \mathcal{N}_{i}(t)\right.
$$

characterizes the price profile of phone $i$ at slot $t$.

\section{Revenue and Gross Profit Maximization}

During the complete time horizon, the server obtains totally $\mathrm{t}_{\text {end }} \sum_{i \in \mathcal{N}} v_{i}\left(\bar{r}_{i}\right)$ amount of monetary revenue (i.e. global social revenue), by selling the collected mobile sensing date to externa MPSSl users. Here, $\bar{r}_{i}$ represents the average sensing rate of phone $i$ over the time horizon $\left[1, \mathrm{t}_{\mathrm{end}}\right]$, and the revenue function $v_{i}\left(\bar{r}_{i}\right)$ can be any concave (includes linear), differentiable, and non-decreasing function of $\bar{r}_{i}$. The revenue function may differ across mobile phones, depending on specific sensing applications and the Quality of Information (QoI) of sensor data produced by each phone $i$ [12], [29]. Therefore, $v_{i}\left(\bar{r}_{i}\right)$ indicates the time-average contribution level of phone $i$ to the MPSS.

At slot $\mathrm{t}_{\mathrm{end}}$, the server computes $v_{i}\left(\bar{r}_{i}\right)$ and makes a payment $\alpha \mathrm{t}_{\text {end }} v_{i}\left(\bar{r}_{i}\right)$ to each phone $i$ (shown in Fig. 1), where the system parameter $0<\alpha \leq 1$ is the percentage of the global social revenue $t_{\text {end }} \sum_{i \in \mathcal{N}} v_{i}\left(\bar{r}_{i}\right)$ that is allocated to all phones. As a result, the time-average gross profit of each phone user is given by:

$$
\varphi_{i}=\alpha v_{i}\left(\bar{r}_{i}\right)-\overline{\operatorname{cost}}_{i}, \forall i \in \mathcal{N}
$$

where $\overline{\operatorname{cost}}_{i}$ is the time-average of $\operatorname{cost}_{i}(t)$ over time horizon $1 \leq t \leq \mathrm{t}_{\text {end }}$.

We call the time-average aggregated gross profit of all mobile phones $\sum_{i \in \mathcal{N}} \varphi_{i}$, as the global gross profit. The MPSS aims to maximize the global gross profit by solving the following finite-horizon stochastic problem:

$$
\begin{aligned}
& \underset{\boldsymbol{x}_{i}(t), i \in \mathcal{N}}{\operatorname{maximize}} \quad \Phi=\sum_{i \in \mathcal{N}} \varphi_{i} \\
& \text { s.t. } \\
& r_{i}(t)<\mathrm{r}_{\max }, \quad i \in \mathcal{N} 1 \leq t \leq \mathrm{t}_{\mathrm{end}} \\
& f_{i, j}(t) \leq \mu_{i, j}(t), \quad i \in \mathcal{N}, j \in \mathcal{N}_{i}(t), 1 \leq t \leq \mathrm{t}_{\mathrm{end}} \\
& \bar{r}_{i}+\bar{f}_{i}^{\text {in }}-\bar{f}_{i}^{\text {out }}=0, i \in \mathcal{N}
\end{aligned}
$$

where $\bar{f}_{i}^{\text {in }}$ and $\bar{f}_{i}^{\text {out }}$ are the time-averages of $f_{i}^{\text {in }}(t)$ and $f_{i}^{\text {out }}(t)$ over time horizon $1 \leq t \leq \mathrm{t}_{\mathrm{end}}$ respectively. Constraint (10) states the flow conservation law, i.e. the average 
total incoming and outgoing data rate should be equal for each phone. This constraint also ensures that the server will know the average sensing rate $\bar{r}_{i}$ for each phone $i \in \mathcal{N}$ at slot $t_{\text {end }}$. Section III will develop OptMPSS, an optimal distributed solution to problem (7)-(10). However, because each phone owner $i$ is only interested in maximizing her own profit $\varphi_{i}$ rather than the global gross profit of the system $\Phi$, the optimal solution to problem (7)-(10) cannot be implemented without proper incentivization mechanism that encourages phone owners to apply the OptMPSS. All variables regarding the incentivization mechanism such as net profit will be defined in Section IV.

\section{E. Objective}

The objective of this paper is to develop an algorithm that can achieve the following desired properties:

1. Global Gross Profit Optimality. The algorithm should be the optimal solution to problem (7)-(10).

2. Adaptiveness. The algorithm should be adaptive to all possible dynamic network states, including time-varying and heterogeneous sensing and transmission cost across phones; wireless link qualities; and network connectivity (e.g. including extremely dense networks where all phones can always communicate with each other, to extremely sparse networks where short-range communication is rare or not available).

3. No Prediction Requirement. The desired algorithm is based on the current system state only, and does not require the prediction of any future MPSS information.

4. Distributed and Real-time Operations. The computational and communication overheads of the algorithm should be lightweight for real-time operations of each phone.

5. Individual Rationality. Each participating phone user should obtain a non-negative net profit, which is formally defined in Equation (18).

6. Server Profitability. The server $S$ should not incur a deficit, which means a non-negative server profit (formally defined in Equation (19)) should be achieved.

7. Incentive Compatibility. Adopting the action suggested by the proposed algorithm should be the best strategy for each phone user, regardless others' actions. An important corollary of incentive compatibility is that using hybrid cellular and (opportunistic) short-range communications will always result in a same or increased net profit for each phone, compared with using the cellular communications alone.

\section{The OptMPSS ALgORITHM}

In this section, we develop an fully distributed algorithm, OptMPSS to optimize global gross profit (7), by controlling the action $\boldsymbol{x}_{i}^{\top}(t)$ of each phone $i \in \mathcal{N}$ at every slot $1 \leq t \leq$ $\mathrm{t}_{\text {end }}$ : its sensing rate $r_{i}(t)$ and the data forwarding rate $f_{i, j}(t)$ to each of its temporary neighbors $j \in \mathcal{N}_{i}(t)$. Initially, in this section, we assume that all phone users are willing to truthfully implement the OptMPSS algorithm. We will relax this assumption in later sections.

\section{A. Distributed Operations of OptMPSS}

At each slot $1 \leq t \leq t_{\text {end }}$, each phone $i \in \mathcal{N}$ operates as follows:

1. Sensing Rate Control. Phone $i$ sets its sensing rate $r_{i}(t)$ as

$$
r_{i}(t)=\min \left(\mathrm{r}_{\max }, \alpha v_{i}^{\prime-1}\left(\frac{Q_{i}(t)+V p_{i}^{s}(t)}{V}\right)\right)
$$

where $v_{i}^{\prime-1}()$ represents the inverse function of revenue function $v_{i}()$ 's first derivative, and $V>0$ is a system parameter defined by the server.

2. Opportunistic Routing and Data forwarding. Phone $i$ computes a weight $w_{i, j}(t)$ for each temporary neighbors $j \in$ $\mathcal{N}_{i}(t)$ as

$$
w_{i, j}(t)=\left(Q_{i}(t)-Q_{j}(t)\right) \mu_{i, j}(t)-V p_{i, j}^{t}(t)
$$

Based on $w_{i, j}(t), i$ sets the forwarding rate $f_{i, j}(t)$ for each of its temporary neighbor $j \in \mathcal{N}_{i}(t)$ as:

$$
f_{i, j}(t)= \begin{cases}\mu_{i, j}(t) & \text { if } w_{i, j}(t)>0 \\ 0 & \text { otherwise }\end{cases}
$$

Remark 1. Since every node $i \in \mathcal{N}$ requires only the information of its temporary neighbors in $\mathcal{N}_{x}(t)$, OptMPSS algorithm is fully distributed. In addition, OptMPSS requires current knowledge of the network only for slot $t$ and does not require any future knowledge after slot $t$. At each slot, each phone broadcasts a one-hop beacon message to communicate its queue backlog to its current temporary neighbors and performs simple arithmetic calculations. Therefore, the per slot per node communication of OptMPSS is $O(1)$ with respect to the network size $|\mathcal{N}|$.

\section{B. Asymptotical Optimality}

To prove the optimality of OptMPSS, We divide the time horizon of the MPSS, $1 \leq t \leq t_{\text {end }}$, into $K$ successive frames with size $T$ slots (i.e. $t_{\text {end }}=K T$ ). We assume that there exists an ideal algorithm operating at the first slot of each frame $t=(k-1) T+1,1 \leq k \leq K$, which can obtain full information regarding the dynamics of the MPSS for future $T$ slots (which is impossible in practice). Based on future knowledge, the ideal algorithm solves problem (7)-(10) over each frame $[(k-1) T+1, k T], 1 \leq k \leq K$ rather than the whole horizon $\left[1, t_{e n d}\right]$. Note that when $T=t_{\text {end }}$, the ideal algorithm becomes the optimal solution of the original problem (7)-(10) . Let $\Phi^{\text {ideal }}(k, T)$ denote the optimal global gross profit computed by the ideal algorithm over each frame $1 \leq k \leq K$.

Theorem 1. The time-average global gross profit computed by OptMPSS satisfies:

$$
\Phi^{\text {OptMPSS }} \geq \frac{1}{K} \sum_{k=1}^{K} \Phi^{\text {ideal }}(k, T)-\frac{M T}{V}
$$

where $M=|\mathcal{N}|\left(\mathrm{r}_{\max }+\mu_{\max }\right)^{2} / 2$ is a constant value.

Proof. Theorem 1 can be proved by using sample-path based 
Lyapunov optimization theory. Due to page limits, we present the details this proof in appendix (https://db.tt/NBeUB9DZ).

Inequality (14) shows that parameter $V$ can be set as large as desired to force $M T / V$ to be arbitrarily small. Specifically, Theorem 1 also demonstrates that when $T=t_{\text {end }}$, the optimal average global gross profit can be asymptotically achieved by OptMPSS, as $V \rightarrow \infty$.

We can see that OptMPSS manages to attain the desired properties 1-4, listed in Subsection II-E. We will discuss how to achieve the other properties in later sections.

\section{Mechanism Design for Faithful MPSS}

A key property of MPSS is that all parameters local to each phone are private and not observable to other phones and the server. Consequently, this provide the phone users with the opportunity to subvert the system by miscommunicating their local parameters. In this section, we briefly discuss algorithmic mechanism design [15], which studies faithful implementation of an intended algorithm in a system with a center and a set of individuals with private parameters. MPSS can be viewed as such a system where the center is a server $S$ and the individuals are mobile phones, and we aim to design a mechanism to faithfully implement the intended OptMPSS algorithm.

\section{A. Centralized Mechanism Design}

Although we focus on distributed mechanism design, for readability, we first discuss direct revelation (centralized) mechanisms [27] in the context of MPSS.

1) Efficient Social Decision: For each phone $i$, define its private type (parameters) as

$$
\theta_{i}=\left(\boldsymbol{p}_{i}(t), \mu_{i, j}(t), j \in \mathcal{N}_{i}(t), 1 \leq t \leq \mathrm{t}_{\text {end }}\right) \in \Theta_{i}
$$

where $\Theta_{i}$ represents the set of all possible types $\theta_{i}$. Denote the private types of all phones as $\boldsymbol{\theta}=\left(\theta_{1}, \ldots, \theta_{|\mathcal{N}|}(t)\right) \in$ $\left(\Theta_{1} \times, \ldots \times \Theta_{|\mathcal{N}|}\right)=\boldsymbol{\Theta}$, where the type space $\Theta$ represents the set of all possible $\theta$.

Let $\mathbf{x}(\boldsymbol{\theta})$ represent the joint rate control and routing decisions of the MPSS during the whole time horizon $1 \leq t \leq$ $t_{\text {end }}$.

$$
\begin{aligned}
\mathbf{x} & =\left(\boldsymbol{x}_{i}(t), i \in \mathcal{N}, 1 \leq t \leq \mathrm{t}_{\text {end }}\right) \\
& =\left(r_{i}(t), f_{i, j}(t), i \in \mathcal{N}, j \in \mathcal{N}_{i}(t), 1 \leq t \leq \mathrm{t}_{\text {end }}\right) \\
& \in \mathcal{X}
\end{aligned}
$$

where $\mathcal{X}$ represents the set of all possible rate control and routing decisions. It is easy to see that the gross profit of each phone $\varphi_{i}, i \in \mathcal{N}$ depends on its private type $\theta_{i}$ and the decision $\mathbf{x}$. Therefore, we can rewrite $\varphi_{i}$ as $\varphi_{i}\left(\mathbf{x}, \theta_{i}\right)$. In mechanism theory, The function $\mathbf{x}: \Theta \rightarrow \mathcal{X}$ is called a social decision.

Definition 1 [Efficient Social Decision] A decision $\mathbf{x}_{\text {opt }}$ is said to be efficient if

$$
\sum_{i \in \mathcal{N}} \varphi_{i}\left(\mathbf{x}_{o p t}, \theta_{i}\right) \geq \sum_{i \in \mathcal{N}} \varphi_{i}\left(\mathbf{x}, \theta_{i}\right)
$$

for all $\boldsymbol{\theta} \in \boldsymbol{\Theta}$ and for all $\mathbf{x} \in \mathcal{X}$. According to Theorem 1 , it can be seen that the sensing rate control and routing decisions made by OptMPSS is the efficient social decision when $V \rightarrow \infty$.

In order to make an efficient social decision $\mathbf{x}(\widehat{\boldsymbol{\theta}})$ in a centralized way, each phone is asked to report its type, denoted as $\widehat{\theta}_{i}$,

$$
\widehat{\theta}_{i}=\left(\widehat{\boldsymbol{p}}_{i}(t), \widehat{\mu}_{i, j}(t), j \in \widehat{\mathcal{N}}_{i}(t), 1 \leq t \leq \mathrm{t}_{\text {end }}\right) \in \Theta_{i}
$$

to the server $S$, where $\widehat{\boldsymbol{\theta}} \in \boldsymbol{\Theta}$ represents the reported types of all phones.

Since each phone user $i$ exhibits strategic behaviors in reality, he or she may be untruthful and report a type value $\widehat{\theta}_{i}$ that is different from the real type (i.e. $\hat{\theta}_{i} \neq \theta_{i}$ ), in order to derive an alternative social decision $\mathbf{x}^{\prime}(\widehat{\boldsymbol{\theta}})$ that results in a better gross profit $\varphi_{i}\left(\mathbf{x}^{\prime}(\widehat{\boldsymbol{\theta}}), \theta_{i}\right)>\varphi_{i}\left(\mathbf{x}_{\text {opt }}, \theta_{i}\right)$.

2) Tax, Subsidy, Net Profit, and Server Profit: In order to make an efficient social decision, server $S$ introduces a monetary transfer function $\lambda: \Theta \rightarrow \mathbb{R}^{|\mathcal{N}|}$

$$
\boldsymbol{\lambda}(\widehat{\boldsymbol{\theta}})=\left(\lambda_{1}(\widehat{\boldsymbol{\theta}}), \ldots, \lambda_{|\mathcal{N}|}(\widehat{\boldsymbol{\theta}})\right)
$$

to encourage the phone users to report their true types. Based on the announcement of a phone $i$ 's type $\widehat{\theta}_{i}(t)$, the function $\lambda_{i}\left(\widehat{\theta}_{i}\right)$, where this is negative this represents a tax that is imposed on phone $i$, or where positive a subsidy is paid to $i$. The combined social decision and monetary transfer function $(\mathbf{x}(\widehat{\boldsymbol{\theta}}), \boldsymbol{\lambda}(\widehat{\boldsymbol{\theta}}))$ is referred to as the social choice function [14]:

$$
g: \Theta \rightarrow \mathcal{X} \times \mathbb{R}^{|\mathcal{N}|}
$$

As a result, the net profit of each phone user is defined as

$$
u_{i}\left(\theta_{i}, \mathbf{x}(\widehat{\boldsymbol{\theta}}), \lambda_{i}(\widehat{\boldsymbol{\theta}})\right)=\varphi_{i}\left(\mathbf{x}(\widehat{\boldsymbol{\theta}}), \theta_{i}\right)+\lambda_{i}(\widehat{\boldsymbol{\theta}})
$$

In our MPSS model, the time-average server profit $u_{S}$ can be formally defined as

$$
u_{S}=(1-\alpha) \sum_{i \in \mathcal{N}} v_{i}\left(\bar{r}_{i}\right)-\sum_{i \in \mathcal{N}} \lambda_{i}(\widehat{\boldsymbol{\theta}})
$$

3) VCG Mechanisms: A direct revelation mechanism is defined as $(g, \boldsymbol{\Theta})$, with a strategy (type) space $\boldsymbol{\Theta}$ and social choice function $g$. A mechanism defines a non-cooperative game with incomplete information as each phone has no knowledge of the types of other phones.

Definition 2 [Incentive Compatibility] A direct revelation mechanism $g(\boldsymbol{\theta})$ is dominant strategy incentive compatible, if the reported $\theta_{i}(t)$ is a dominant strategy for each phone $i \in \mathcal{N}$ :

$$
u_{i}\left(g\left(\theta_{i}, \theta_{-i}\right), \theta_{i}\right) \geq u_{i}\left(g\left(\widehat{\theta}_{i}, \theta_{-i}\right), \theta_{i}\right), \forall \theta_{-i}, \theta_{i}, \widehat{\theta}_{i} \neq \theta_{i}
$$

, where $\theta_{-i}(t)$ represents the types of all other phones $j \in$ $\mathcal{N}-\{i\}$. In this case we say the social choice function is implemented in ex-post Nash equilibria.

The Vickrey-Clarke-Groves (VCG) mechanism is wellknown for the desired property of incentive compatibility [14]. The social decision rule of the VCG mechanism is given by

$$
\mathbf{x}^{v c g}(\widehat{\boldsymbol{\theta}})=\underset{\mathbf{x}(\widehat{\boldsymbol{\theta}}) \in \mathcal{X}}{\arg \max } \sum_{i \in \mathcal{N}} \varphi_{i}\left(\mathbf{x}(\widehat{\boldsymbol{\theta}}), \widehat{\theta}_{i}\right)
$$


and the monetary transfer function of each phone $i \in \mathcal{N}$ is:

$$
\lambda_{i}^{v c g}\left(\widehat{\theta}_{i}\right)=\underbrace{\sum_{j \neq i} \varphi_{j}\left(\mathbf{x}^{v c g}(\widehat{\boldsymbol{\theta}}), \widehat{\theta_{j}}\right)}_{(\mathrm{a})}-\underbrace{\max _{\mathbf{x}_{-i} \in \mathcal{X}_{-i}} \sum_{j \neq i} \varphi_{j}\left(\mathbf{x}_{-i}, \widehat{\theta}_{j}\right)}_{(\mathrm{b})}
$$

where the term (a) corresponds to the gross profit of all phones excluding $i$ (i.e. all phones in $\mathcal{N}-\{i\}$ ) when an efficient social decision has been made, and term (b) represents the maximum global gross profit achievable for all phones in $\mathcal{N}-\{i\}$, without $i$ 's presence in the MPSS. Therefore, the monetary transfer $\lambda_{i}^{v c g}\left(\widehat{\theta}_{i}\right)$ represents the impact (either loss or increase) in value that is imposed on all other individuals (i.e. marginal social impact) due to the social decision that has been updated resulting from $i$ 's presence in the MPSSs.

\section{B. Distributed Mechanism Design}

A distributed mechanism $d M=(h, \Pi, A)$ [13], [27] defines an outcome (i.e. decision and taxing) function $h=$ $\left(h_{\mathbf{x}}, h_{\boldsymbol{\lambda}}\right)$, a feasible strategy space $\Pi=\left(\Pi_{1} \times \ldots \times \Pi_{|\mathcal{N}|}\right)$ (i.e. all possible sensing rate control and routing strategies in our MPSS model), and intended distributed algorithm (i.e. strategy) $A=\left(A_{1}, \ldots, A_{|\mathcal{N}|}\right)$, such as our OptMPSS algorithm. A strategy $s=\left(s_{1}, \ldots, s_{|\mathcal{N}|}\right) \in \Pi$ is a mapping from type space to distributed actions including message transmission and computation. The social choice function can be viewed as $g=h \circ s(\boldsymbol{\Theta})=(\mathbf{x}, \boldsymbol{\lambda})$. Fig. 2 illustrates the relationship between directed and distributed mechanisms.

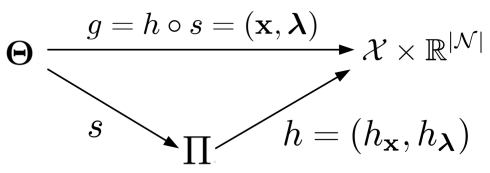

Fig. 2. The relations between direct and distributed mechanisms.

Definition 3 [Faithful Implementation]. A distributed mechanism $d M=(h, \Pi, A)$ is an faithful implementation of the social choice function $g=h \circ A(\boldsymbol{\theta}) \in \mathcal{X} \times \mathbb{R}^{|\mathcal{N}|}$, when the intended distributed algorithm (i.e. strategies for phones) $A=\left(A_{1}, \ldots, A_{|\mathcal{N}|}\right)$ is an ex post Nash equilibrium.

$$
u_{i}\left(h\left(A_{i}\left(\theta_{i}\right), A_{-i}\left(\theta_{-i}\right), \theta_{i}\right) \geq u_{i}\left(h\left(A_{i}^{\prime}\left(\theta_{i}\right), A_{-i}\left(\theta_{-i}\right), \theta_{i}\right)\right.\right.
$$

for all $i \in \mathcal{N}, A_{i}^{\prime} \neq A_{i}, \theta_{i}, \theta_{-i}$. In this case, the distributed mechanism $d M=(h, \Pi, A)$ is also said to be incentive compatible.

In our MPSS model, the intended distributed algorithm $A$ is OptMPSS. Due to the asymptotical optimality of our OptMPSS, we define the asymptotic incentive compatibility as follows:

Definition 4 [Asymptotic Incentive Compatibility]. A distributed mechanism $d M=(h, \Pi, A)$ is asymptotically incentive compatible, if

$u_{i}\left(h\left(A_{i}\left(\theta_{i}\right), A_{-i}\left(\theta_{-i}\right), \theta_{i}\right) \geq u_{i}\left(h\left(A_{i}^{\prime}\left(\theta_{i}\right), A_{-i}\left(\theta_{-i}\right), \theta_{i}\right)-\varepsilon(V)\right.\right.$ for all $i \in \mathcal{N}, A_{i}^{\prime} \neq A_{i}, \theta_{i}, \theta_{-i}$, where $\varepsilon(V)>0$ and $\varepsilon(V) \rightarrow 0$ as $V \rightarrow \infty$.

\section{The BMT AlgorithM}

By applying distributed mechanism design to OptMPSS, we develop BMT, an on-line and fully distributed algorithm that calculates the marginal social impact (for computing the VCG tax) of each phone. in parallel with the operations of OptMPSS.

\section{A. Distributed Operations of BMT}

During the complete time horizon $1 \leq t \leq \mathrm{t}_{\mathrm{end}}$, the BMT algorithm operates as follows:

1. Initialization. At beginning of slot $t=1$.

- The server: $S$ broadcasts the set $\mathcal{N}$, revenue function $\alpha v_{i}()$, and system parameters $V$ and $\mathrm{r}^{\max }$ to each phone $i \in \mathcal{N}$.

- Mobile phones: Besides storing its data queue holding data, each phone $i$ initializes a virtual queue length (a non-negative integer number) $Q_{i}^{-j}(t)$ for each of all other phones $j \in \mathcal{N}, j \neq i$, where $Q_{i}^{-j}(t)$ means the queue length of phone $i$ without phone $j$ 's presence. The initial lengths of all virtual queues are set as zero.

2. At each slot $1 \leq t \leq \mathrm{t}_{\mathrm{end}}$

Distributed Sensing and Routing. Each phone $i \in \mathcal{N}$ adopts the OptMPSS algorithm for optimal distributed sensing and data forwarding.

Distributed Marginal Social Impact Computation. In parallel, each phone $i \in \mathcal{N}$ computes the virtual sensing rate $r_{i}^{-j}(t)$ and the virtual cost $\operatorname{cost}_{i}^{-j}(t)$ for each of all other phones $j \in \mathcal{N}, j \neq i$ based on the corresponding virtual queue length $Q_{i}^{-j}(t)$,

$$
r_{i}^{-j}(t)=\min \left(\mathrm{r}_{\max }, \alpha v_{i}^{\prime-1}\left(\frac{Q_{i}^{-j}(t)+p_{i}^{s}(t)}{V}\right)\right)
$$

and

$$
\operatorname{cost}_{i}^{-j}(t)=p_{i}^{s}(t) r_{i}^{-j}(t)+\sum_{k \in \mathcal{N}_{i}(t), k \neq j} f_{i, k}^{-j} c_{i, k}^{t}(t)
$$

where the virtual forwarding rate is

$$
f_{i, k}^{-j}(t)= \begin{cases}\mu_{i, k}(t) & \text { if } w_{i, k}^{-j}(t)>0 \\ 0 & \text { otherwise }\end{cases}
$$

where for each $k \in \mathcal{N}_{i}(t), k \neq j$, the virtual weight is

$$
w_{i, k}^{-j}(t)=\left(Q_{i}^{-j}(t)-Q_{k}^{-j}(t)\right) \mu_{i, k}(t)-V p_{i, k}^{t}(t)
$$

The virtual queue lengths $Q_{i}^{-j}(t), \forall j \in \mathcal{N}-\{i\}$ are updated as

$$
\begin{aligned}
Q_{i}^{-j}(t+1)= & \left|Q_{i}^{-j}(t)-\sum_{k \in \mathcal{N}_{i}(t)} f_{i, k}^{-j}(t)\right|_{+}+r_{i}^{-j}(t) \\
& +\sum_{k \in \mathcal{N}_{i}(t)} f_{k, i}^{-j}(t)
\end{aligned}
$$


The average virtual sensing rates and virtual costs for all $j \neq$ $i, j \in \mathcal{N}$ are updated as

$$
\begin{aligned}
& \bar{r}_{i}^{-j}=\left(r_{i}^{-j}+(t-1) \bar{r}_{i}^{-j}\right) / t \\
& \overline{\operatorname{cost}}_{i}^{-j}=\left(\operatorname{cost}_{i}^{-j}+(t-1) \overline{\operatorname{cost}}_{i}^{-j}\right) / t
\end{aligned}
$$

\section{At slot $t=\mathrm{t}_{\mathrm{end}}$.}

- Each phone $i$ reports the average virtual sensing rates $\bar{r}_{i}^{-j}$ and virtual cost $\overline{\operatorname{cost}}_{i}^{-j}$ for all $j \in \mathcal{N}, j \neq i$ to the server.

- The server The server can compute the VCG tax

$$
\begin{aligned}
\lambda_{i}^{v c g}= & \sum_{j \in \mathcal{N}-\{i\}}\left(\alpha v_{j}\left(\bar{r}_{j}^{-i}\right)-\overline{\operatorname{cost}}_{j}^{-i}\right) \\
& -\sum_{j \in \mathcal{N}-\{i\}}\left(\alpha v_{j}\left(\bar{r}_{j}\right)-\overline{\operatorname{cost}}_{j}\right)
\end{aligned}
$$

of each phone $i \in \mathcal{N}$. Finally, the server makes a payment of $\mathrm{t}_{\text {end }}\left(\alpha v_{i}\left(\bar{r}_{i}\right)+\lambda_{i}^{v c g}\left(\widehat{\theta}_{i}\right)\right)$ to phone $i$.

From a global view of point, the BMT algorithm runs in total one real and $|\mathcal{N}|$ virtual OptMPSS algorithms in parallel during the time horizon of the MPSS: the real OptMPSS algorithm makes the actual sensing rate and routing decisions at each slot, while $|\mathcal{N}|$ virtual OptMPSS algorithms simulate $\mathcal{N}$ virtual marginal societies with absence of each phone $i \in \mathcal{N}$ to compute the final tax or subsidy for each phone in a fully distributed way.

Remark 2. It worth noting that the $|\mathcal{N}|-1$ virtual queue lengths maintained in each phone are integer numbers rather than real data packet queues, which results in negligible storage overheads for mobile phones (e.g. only several KB storage overhead for a MPSS with thousands of phones with several GB RAMs). In addition, BMT requires each node to transmit $O(|\mathcal{N}|)$ bytes of information (its real and maintained virtual queue backlogs) to its current neighbors only, while also performing $O(|\mathcal{N}|)$ simple arithmetic calculations. This is still realistic for today's smart phones using short-range radios such as WiFi direct that can achieve up to $250 \mathrm{Mbps}$ data transmission rate. Due to its distributed operations and light overheads, BMT has a great potential to be applied in large-scale MPSS.

\section{B. Asymptotic Incentive Compatibility}

In this subsection, we prove that BMT achieves asymptotic incentive compatibility. We first introduce a new definition and a supportuing lemma.

Definition 5 [Asymptotically Efficient Social Decision] For a distributed mechanism $d M=(h, \Pi, A)$, a social decision $h_{x}(\boldsymbol{\theta})$ made by the suggested algorithm $A$ is said to be asymptotically efficient if

$$
\sum_{i \in \mathcal{N}} \varphi_{i}\left(h_{\mathbf{x}} \circ A(\boldsymbol{\theta}), \theta_{i}\right) \geq \sum_{i \in \mathcal{N}} \varphi_{i}\left(h_{\mathbf{x}} \circ A^{\prime}(\boldsymbol{\theta}), \theta_{i}\right)-\varepsilon(V)
$$

for all $\boldsymbol{\theta} \in \boldsymbol{\Theta}$ and for all $A^{\prime} \in \Pi$, where $\varepsilon(V)>0$ and $\varepsilon(V) \rightarrow 0$ as $V \rightarrow \infty$.

Lemma 1. The social decision made by BMT algorithm, $\boldsymbol{x}^{b m t}$

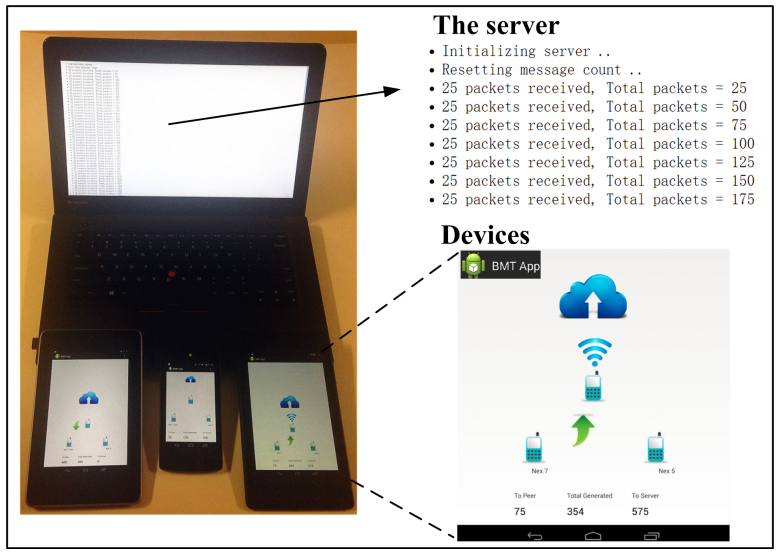

Fig. 3. Experiment Prototype Illustration.

is asymptotically efficient.

Proof. Since the distributed social decision (e.g. sensing rate control and routing decisions) made by BMT is the same as that of OptMPSS, this Lemma obviously holds when $\varepsilon(V)=M T / V$ and frame size $T=\mathrm{t}_{\text {end }}$, according to Theorem 1.

Theorem 2. BMT achieves asymptotic incentive compatibility.

Proof. We prove Theorem 2 by contradiction. Consider a distributed mechanism $d M=\left(h, \Pi, A^{b m t}\right)$, where $A^{b m t}=$ $\left(A_{1}^{b m t}, \ldots, A_{|\mathcal{N}|}^{b m t}\right)$ is the distributed strategy of each phone allocated by BMT algorithm. Suppose that BMT is not asymptotically incentive compatible, i.e. $\exists i \in \mathcal{N}, A_{i}^{\prime} \neq A_{i}^{b m t}$ such that

$$
\begin{aligned}
& \begin{array}{l}
u_{i}\left(h\left(A_{i}^{b m t}\left(\theta_{i}\right), A_{-i}^{b m t}\left(\theta_{-i}\right), \theta_{i}\right)+\varepsilon(V)\right. \\
={ }_{(a)} \varphi_{i}\left(h_{\mathbf{x}}\left(A_{i}^{b m t}\left(\theta_{i}\right), A_{-i}^{b m t}\left(\theta_{-i}\right)\right), \theta_{i}\right) \\
\quad+h_{\lambda_{i}}\left(A_{i}^{b m t}\left(\theta_{i}\right), A_{-i}^{b m t}\left(\theta_{-i}\right)\right)+\varepsilon(V) \\
={ }_{(b)} \sum_{i \in \mathcal{N}} \varphi_{i}\left(h_{\mathbf{x}}\left(A_{i}^{b m t}\left(\theta_{i}\right), A_{-i}^{b m t}\left(\theta_{-i}\right)\right), \theta_{i}\right) \\
\quad-\max \sum_{j \neq i} \varphi_{j}+\varepsilon(V) \\
<\sum_{i \in \mathcal{N}} \varphi_{i}\left(h_{\mathbf{x}}\left(A_{i}^{\prime}\left(\theta_{i}\right), A_{-i}^{b m t}\left(\theta_{-i}\right), \theta_{i}\right)-\max \sum_{j \neq i} \varphi_{j}\right. \\
=u_{i}\left(h_{\mathbf{x}}\left(A_{i}^{\prime}\left(\theta_{i}\right), A_{-i}^{b m t}\left(\theta_{-i}\right), \theta_{i}\right)\right.
\end{array}
\end{aligned}
$$

where equalities $(a)$ and $(b)$ follow the definitions of net profit and VCG tax respectively. It can be seen that inequality (26) implies that

$\sum_{i \in \mathcal{N}} \varphi_{i}\left(h_{\mathbf{x}} \circ A^{b m t}(\boldsymbol{\theta}), \theta_{i}\right)<\sum_{i \in \mathcal{N}} \varphi_{i}\left(h_{\mathbf{x}} \circ A^{\prime}(\boldsymbol{\theta}), \theta_{i}\right)-\varepsilon(V)$

where $A^{\prime}=\left(A_{1}^{b m t}, \ldots, A_{i}^{\prime}, \ldots, A_{|\mathcal{N}|}^{b m t}\right)$. This contradicts the asymptotically social efficiency of BMT, i.e. Lemma 1.

\section{Evaluation}

In this section, we evaluate the performance of the BMT algorithm via both prototype experiments and simulations 


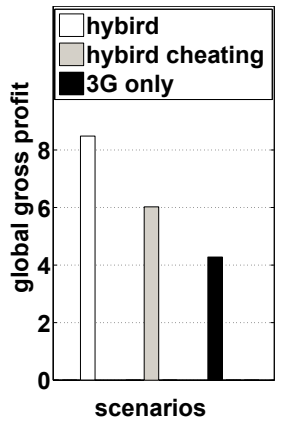

(a)

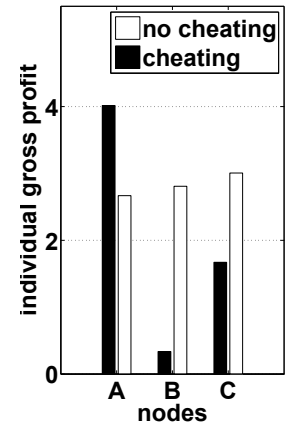

(b)

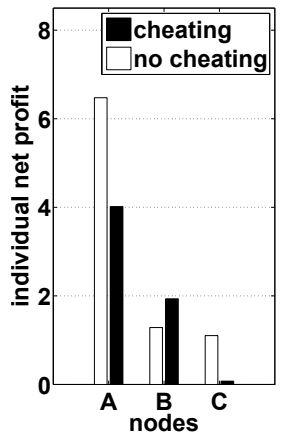

(c)
Fig. 4. Experiment results: (a) time-average global gross profits for the three experiments. (b) and (c) shows the impact of device A's cheating action on the time-average individual gross and net profits of every device respectively.

using real human mobility traces.

\section{A. Experiments Based on Android Device}

We implemented the BMT algorithm in Android OS 4.3, and developed an application called BMT App. We constructed a proof-of-concept MPSS with three WiFi-direct enabled Android devices (i.e. a Nexus 5 phone and two Nexus 7 tablets) and a server implemented in NODE.JS (http://nodejs.org/), as shown in Fig.3. The duration of a slot was set as two seconds and the duration of each experiment was 10 minutes. At the each slot, the BMT App run a discovery phase to update the temporary neighbor table (e.g. the server and nearby devices), and then performed the sensing, routing, and marginal social impact computation tasks as defined by the BMT algorithm. Each device was held by a researcher moved around our lab.

We use the revenue function $v_{i}\left(\bar{r}_{i}\right)=4 \ln \left(1+\bar{r}_{i}\right)$ for each $i \in \mathcal{N}$, and set the system parameters $V=100$ and $\alpha=0.5$. We use WiFi direct as the short-range radios. The channel capacities of all wireless radios are set at 25 packets per slot. The sensing prices and transmission prices (in credits per data packet) for the data sent by $\mathrm{WiFi}$ direct were set as 0.1 , for all three Android devices $A, B$, and $C$. The $3 \mathrm{G}$ transmission prices of $A, B$ and $C$ were set as $0.1,1$, and 1.5 respectively ${ }^{1}$.

As shown in Fig.4 (a), the time-average global gross profit of MPSS using hybrid $3 \mathrm{G}$ and $\mathrm{WiFi}$ direct communications is approximately twice of that using $3 \mathrm{G}$ radios alone, which demonstrates that significant performance improvement can be achieved by using hybrid cellular and short-range radio.

In addition, we also evaluated the incentive compatibility of BMT. Since device $A$ had a much lower $3 \mathrm{G}$ prices than $B$ and $C$, the routing decisions made by BMT would require $A$ to relay the sensor data packets collected by $B$ and $C$ (when $A$ passed them) to the server, in order to maximize the global gross profit. However, this would result in an individual gross profits reduction for $A$ (due to the relaying cost), and therefore (the owner of) $A$ may not want to faithfully adopt the distributed routing actions suggested by BMT. To check

\footnotetext{
${ }^{1}$ This implies that $A$ may have an unlimited mobile data budget, while $B$ and $C$ may adopt a limited monthly contract or 'pay as you go' tariff rate.
}

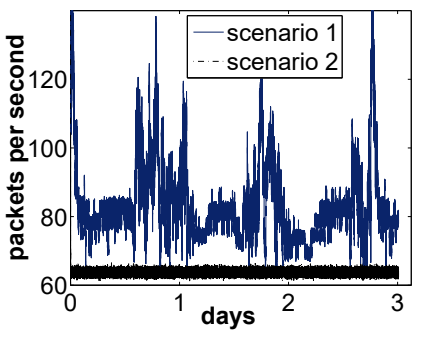

(a) dynamic throughput

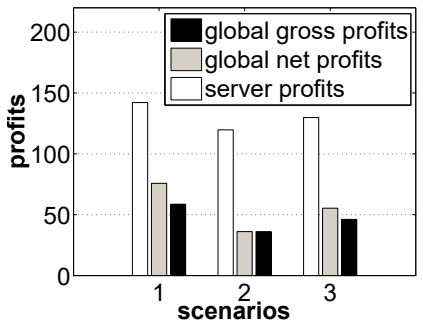

(c) global profits (b) average throughput

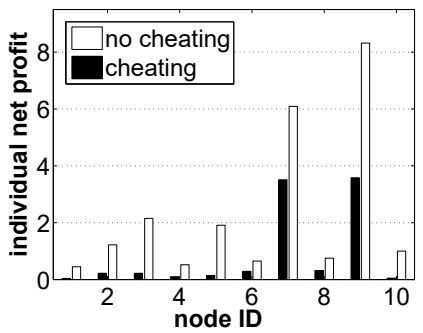

(d) incentive compatibility

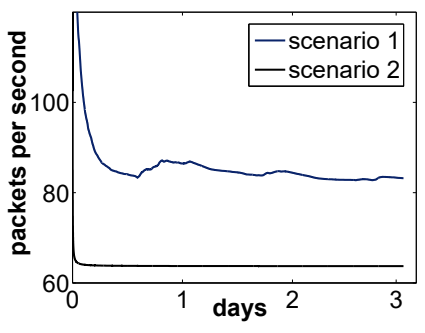

Fig. 5. Simulation results of BMT.

whether BMT can avoid this, we mimic a quite intuitive cheating behavior for $A$, i.e. disabling its WiFi direct radio.

Fig. 4 (b) shows that the individual gross profit for each device before and after $A$ 's cheating action. It can be seen that this untruthful behavior can indeed improve $A$ 's individual gross profit, but results in a significant degeneration of global gross profit for the whole system, as shown in Fig. 4(a). As shown in Fig.4 (c), however, $A$ eventually missed the opportunity of obtaining approximately $36 \%$ more net profit due to cheating. This means that $A$ would be better off relaying sensor data from other devices than misinforming the network. This demonstrates that in practice BMT can achieve the highly desired incentive compatibility property. In addition, the server profit and net profit of each device and server profit were positive in all experiments (These results are not plotted due to page limits), which demonstrates that BMT can achieve individual rationality and server profitability in practice.

\section{B. Trace-driven Simulations}

To evaluate the practical performance of BMT at scale, we established simulations using the real human trace collected from Infocom05 (41 nodes for 3 days) [17]. In all simulations, each phone has both $3 \mathrm{G}, \mathrm{WiFi}$, and $\mathrm{WiFi}$ direct radios. When a phone meets a free $\mathrm{WiFi}$ router, it sends data through the WiFi radio rather than $3 \mathrm{G}$. In each simulation, a power-law distributed random variable was assigned to each phone to simulate the heterogeneous free WiFi access probability across phones, observed from real human mobility traces [30], [31]. The sensing and WiFi/Wifi-direct transmission prices for each phone were dynamically set between 0 and 0.01 for each slot (representing channel quality variation) at each slot, while $3 \mathrm{G}$ transmission price of each phone was randomly set between 0.1 and 0.5 at the beginning of each simulation and remained constant over slots. We set $r_{\max }=5$ and the duration of a 
slot as one second. All other simulation parameters were set the same as in the prototype experiment represented earlier.

We again run three simulations: MPSS with all wireless radios (scenario 1), MPSS without WiFi direct (scenario 2), and cheating actions in MPSS with all wireless radios (scenario 3 ), where the users of phone 1 to 10 try to hide their WiFi direct abilities. Fig. 5 (a) and (b) show the dynamic and timeaverage throughput (i.e. total sensing data packets produced by all phones at each slot) of MPSS respectively, which demonstrate that the BMT algorithm is adaptive to network dynamics (e.g. mobility) and manages to converge to the timeaverage optimal. In addition, Figure 5 (c) and (d) demonstrate that using multi-hop opportunistic short-range communications can significantly improve network performance and BMT can achieve incentive compatibility, individual rationality, and server profitability in practice.

\section{CONCLUSION}

In this paper we investigate a cost-effective data collection solution to Mobile Phone Sensing Systems (MPSS) that utilize hybrid cellular and opportunistic short-range wireless communications. We formulate a stochastic optimization problem for mobile sensor data collection, and develop OptMPSS, a scalable joint sensing rate control and routing algorithm to solve the formulated optimization problem in a fully distributed and scalable way. In order to encourage phone users to faithfully apply the OptMPSS algorithm's control suggestions, we propose BMT, a joint networking and taxing scheme, based on combing Lyapunov stochastic optimization and distributed mechanism design theories. We prove that BMT achieves asymptotical optimality and incentive compatibility.

In order to evaluate the practical performance of BMT, we developed BMT App, an Android application that implements BMT algorithm for WiFi-direct-enabled devices. Trough experiments and trace-driven simulations, we demonstrate that BMT can efficiently exploit low-cost short-range communications, which significantly improves the global gross profit of the MPSS (around 100\%). Evaluation results show that the networking actions suggested by BMT are the best choice for each individual phone user. In addition, evaluation results also demonstrate that our approach can achieve individual rationality and server profitability in practice.

\section{REFERENCES}

[1] R. K. Ganti, F. Ye, and H. Lei, "Mobile crowdsensing: current state and future challenges," IEEE Commun. Mag., vol. 49, no. 11, pp. 32-39, 2011.

[2] N. D. Lane, E. Miluzzo, H. Lu, D. Peebles, T. Choudhury, and A. T. Campbell, "A survey of mobile phone sensing," IEEE Communications. Mag., vol. 48, no. 9, pp. 140-150, 2010.

[3] R. K. Rana, C. T. Chou, S. S. Kanhere, N. Bulusu, and W. Hu, "Earphone: an end-to-end participatory urban noise mapping system," in Proc. IPSN, 2010, pp. 105-116.

[4] A. Thiagarajan, L. Ravindranath, K. LaCurts, S. Madden, H. Balakrishnan, S. Toledo, and J. Eriksson, "Vtrack: accurate, energy-aware road traffic delay estimation using mobile phones," in Proc. ACM SenSys.

[5] J. Whipple, W. Arensman, and M. S. Boler, "A public safety application of gps-enabled smartphones and the android operating system," in Proc. IEEE SMC, 2009, pp. 2059-2061.
[6] X. Sheng, J. Tang, and W. Zhang, "Energy-efficient collaborative sensing with mobile phones," in IEEE INFOCOM, 2012, pp. 1916-1924.

[7] L. Wang, D. Zhang, and H. Xiong, "effsense: energy-efficient and cost-effective data uploading in mobile crowdsensing," in Proc. ACM UbiComp, 2013, pp. 1075-1086.

[8] H. Liu, S. Hu, W. Zheng, Z. Xie, S. Wang, P. Hui, and T. Abdelzaher, "Efficient $3 \mathrm{~g}$ budget utilization in mobile participatory sensing applications," in Proc. IEEE INFOCOM, 2013, pp. 1411-1419.

[9] K. K. Rachuri, C. Efstratiou, I. Leontiadis, C. Mascolo, and P. J. Rentfrow, "Metis: Exploring mobile phone sensing offloading for efficiently supporting social sensing applications," in Proc. IEEE PerCom, 2013, pp. 85-93.

[10] J.-S. Lee and B. Hoh, "Sell your experiences: a market mechanism based incentive for participatory sensing," in Proc. IEEE PerCom 2010, 2010, pp. 60-68.

[11] D. Yang, G. Xue, X. Fang, and J. Tang, "Crowdsourcing to smartphones: incentive mechanism design for mobile phone sensing," in Proc. ACM Mobicom, 2012, pp. 173-184.

[12] I. Koutsopoulos, "Optimal incentive-driven design of participatory sensing systems," in Proc. IEEE INFOCOM, 2013, pp. 1402-1410.

[13] T. Tanaka, F. Farokhi, and C. Langbort, "A faithful distributed implementation of dual decomposition and average consensus algorithms," in Proc. IEEE CDC, 2013.

[14] M. O. Jackson, "Mechanism theory," The Encyclopedia of Life Support Systems, 2000.

[15] N. Nisan and A. Ronen, "Algorithmic mechanism design," in Proc. ACM STOC, 1999, pp. 129-140.

[16] L. Georgiadis, M. Neely, M. Neely, and L. Tassiulas, Resource allocation and cross-layer control in wireless networks. Now Pub, 2006.

[17] "http://crawdad.cs.dartmouth.edu/ crawdad/cambridge/haggle/."

[18] T. Luo, H.-P. Tan, and L. Xia, "Profit-maximizing incentive for participatory sensing," in Proc. IEEE Infocom, 2014.

[19] W. Dong, S. Rallapalli, R. Jana, L. Qiu, K. Ramakrishnan, L. Razoumov, Y. Zhang, and T. W. Cho, "ideal: Incentivized dynamic cellular offloading via auctions," in Proc. IEEE INFOCOM, 2013, pp. 755-763.

[20] X. Zhuo, W. Gao, G. Cao, and Y. Dai, "Win-coupon: An incentive framework for $3 \mathrm{~g}$ traffic offloading," in Proc. IEEE ICNP, 2011, pp. 206-215.

[21] K. Lee, J. Lee, Y. Yi, I. Rhee, and S. Chong, "Mobile data offloading: how much can wifi deliver?" in In Proc. CoNEXT, 2010, p. 26.

[22] B. Han, P. Hui, V. A. Kumar, M. V. Marathe, J. Shao, and A. Srinivasan, "Mobile data offloading through opportunistic communications and social participation," IEEE Trans. Mobi. Comput., vol. 11, no. 5, pp. 821-834, 2012.

[23] J. Ryu, V. Bhargava, N. Paine, and S. Shakkottai, "Back-pressure routing and rate control for icns," in Proc. ACM Mobicom, 2010, pp. 365-376.

[24] S. Yang, U. Adeel, and J. A. McCann, "Selfish mules: Social profit maximization in sparse sensornets using rationally-selfish human relays," IEEE J. Sel. Areas Commun., vol. 31, no. 6, pp. 1124-1134, 2013.

[25] M. Alresaini, M. Sathiamoorthy, B. Krishnamachari, and M. J. Neely, "Backpressure with adaptive redundancy (bwar)," in Proc. IEEE INFOCOM, 2012, pp. 2300-2308.

[26] S. Yang, Z. Sheng, J. McCann, and K. Leung, "Distributed stochastic cross-layer optimization for multi-hop wireless networks with cooperative communications," IEEE Trans. Mobi. Comput., vol. 13, no. 10, pp. 2269-2282, 2014.

[27] D. C. Parkes and J. Shneidman, "Distributed implementations of vickreyclarke-groves mechanisms," in Proc. AAMAS, 2004, pp. 261-268.

[28] M. Bakht, M. Trower, and R. H. Kravets, "Searchlight: won't you be my neighbor?" in Proc. ACM Mobicom, 2012, pp. 185-196.

[29] C. H. Liu, P. Hui, J. W. Branch, C. Bisdikian, and B. Yang, "Efficient network management for context-aware participatory sensing," in Proc. IEEE SECON, 2011, pp. 116-124.

[30] S. Yang, X. Yang, C. Zhang, and E. Spyrou, "Using social network theory for modeling human mobility," IEEE Network, vol. 24, no. 5, pp. 6-13, 2010.

[31] A. Chaintreau, P. Hui, J. Crowcroft, C. Diot, R. Gass, and J. Scott, "Impact of human mobility on opportunistic forwarding algorithms," IEEE Trans. Mobi. Comput., vol. 6, no. 6, pp. 606-620, 2007. 